\title{
A rare case of ruptured hydatid cyst presenting as a left upper lobe lung abscess
}

\author{
Pankaj Gholap ${ }^{1, *}$, Sushant Meshram² \\ ${ }^{\mathbf{1}}$ Associate Professor, ${ }^{2}$ Professor and Head, Dept. of Respiratory Medicine, Government Medical College, Nagpur, Maharashtra, \\ India
}

*Corresponding Author:

Email: drpankajgholap@gmail.com

\begin{abstract}
Echinococcosis is caused primarily by one of two species of cestodes. Echinococcus granulosus and Echinococcus multilocularis. Here we present a case history of 18-year-old non-smoker farmer presented to the OPD with a one month history of moderate grade fever, 8 days history of cough with expectoration and a chest x-ray and CT thorax report suggestive of left upper lobe lung abscess. On routine bronchoscopy, the left upper lobe was seen completely obstructed with folded handkerchief shaped white membranous tissue which aroused the suspicion of a ruptured hydatid cyst. On review of the CT report, the lesion was reported to be a ruptured hydatid cyst and managed accordingly.
\end{abstract}

Keyword: Ruptured hydatid cyst, Bronchoscopy, Lung abscess.

\section{Introduction}

Echinococcosis is caused primarily by one of two species of cestodes. Echinococcus granulosus is the dog tapeworm that requires two hosts to complete its life cycle. Dogs or other canines are the definitive hosts and sheep, goats, and other domestic animals are the intermediate hosts which occurs worldwide. $^{1}$ Echinococcus multilocularis which is also called the fox tapeworm causes alveolar echinococcus and has a sylvatic life cycle in which the definitive hosts are foxes and other carnivores, the intermediate hosts being rodents and small mammals. This form of the disease is endemic in the northern hemisphere in Europe, China, and northern Canada/Alaska. Echinococcus multilocularis is the more pathogenic of the two major species although Echinococcus granulosus is the more common of the two. In the normal life cycle, adult worms living in the intestines of the definitive host shed millions of eggs in the stool. On ingestion of an egg by an intermediate host, a larva emerges, penetrates the intestinal mucosa, and migrates through the blood or lymphatics to target organs. The larvae usually migrate to the liver or lung and transform into metacestodes that create vesicles that enlarge over time to form a mature hydatid cyst. The mature hydatid cyst consists of multiple brood capsules and protoscolices. The adult tapeworm develops in the definitive host who ingests the cyst. ${ }^{1-2}$

Humans become incidental intermediate hosts by ingesting eggs on dogs because of improper or lack of hand washing or by ingesting contaminated food or water.

\section{Case History}

A 18-year-old male farmer, non-smoker, nonalcoholic presented to a tertiary care centre in central India in the department of Pulmonary Medicine with chief complaints of moderate grade intermittent fever since 1 month which had resolved since 5 days with medication, generalized weakness and loss of appetite since one month and cough with scanty mucoid expectoration since 8 days. General and Respiratory system examination were unremarkable. The total leukocyte count was $8000 / \mathrm{cmm}$ with $70 \%$ Neutrophils, $28 \%$ Lymphocytes, $1 \%$ Eosinophils and 1\% Monocytes. Sputum gram stain and culture sensitivity report was inconclusive. Haemoglobin was low with $\mathrm{Hb}$ of $9.5 \mathrm{gm} / \mathrm{dl}$. Sputum Acid Fast Bacilli (AFB) smear and Cartridge Based Nucleic Acid Amplification Test (CB-NAAT) for Mycobacterium tuberculosis (MTB) were negative.

The chest $\mathrm{x}$-ray report showed a rounded opacity with air fluid level in left upper lobe? Abscess (Fig. 1 and Fig. 2).

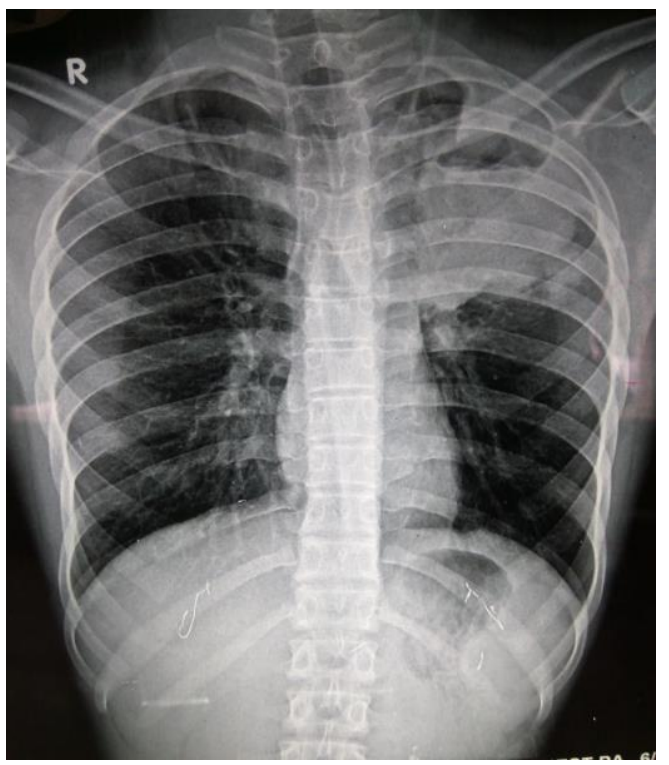

Fig. 1: Chest $x$-ray PA view of left upper lobe cystic lesion with air fluid level 


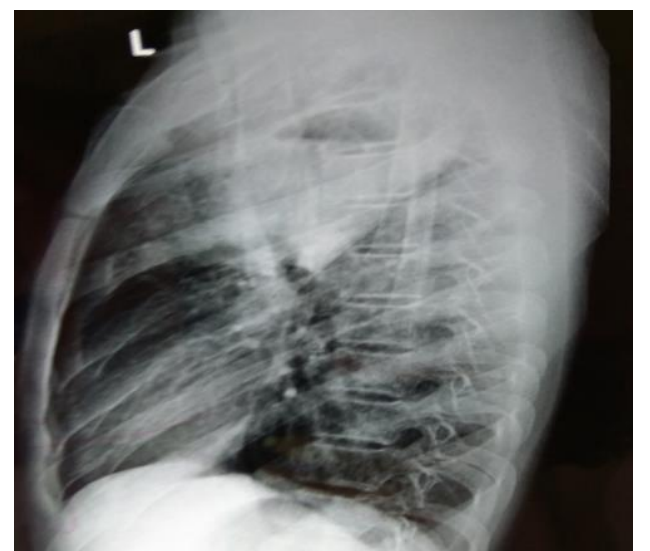

Fig. 2: Left lateral view of left upper lobe hydatid cyst

CT thorax with contrast reported a thick walled cavity with air fluid level involving left upper lobe. Surrounding ill defined heterogeneously enhancing areas of consolidation noted involving the left upper lobe with confluent nodular opacities. Significant volume loss of left upper lobe was also noted. Report concluded that findings were most likely suggestive of infective aetiology with lung abscess (Fig. 3).

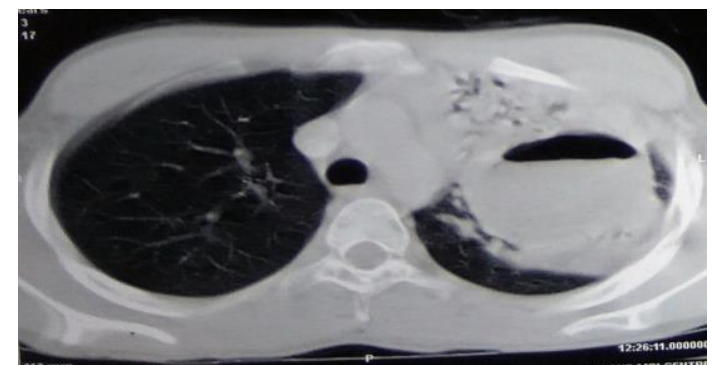

Fig. 3: CT of left upper lobe ruptured hydatid cyst with surrounding consolidation

A video bronchoscopy of the patient was done with a flexible bronchoscope to visualize the left upper lobe. On flexible bronchoscopy the left upper lobe bronchus was seen to be completely obstructed with a folded handkerchief shaped white membranous tissue. This finding on bronchoscopy aroused a suspicion of a ruptured hydatid cyst and a review of the CT thorax report was asked for (Fig. 4).

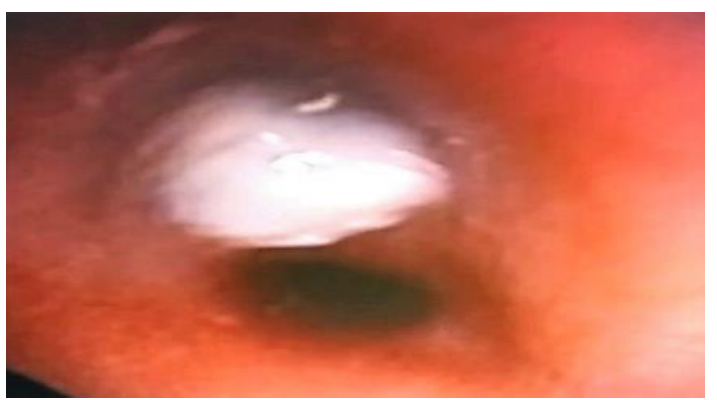

Fig. 4: Bronchoscopic view of left upper lobe completely obstructed with membranes of ruptured hydatid cyst

A reviewed CT thorax report in light of the bronchoscopic findings was changed as a well defined cystic lesion with membranes within with consolidation of the left upper lobe suggestive of infected hydatid cyst.

\section{Management and Outcome}

The patient was initially managed as a case of lung abscess based on the CT report and was started on Inj. Piperacillin and Tazobactum $4.5 \mathrm{mg}$ IV TDS and Inj. Metronidazole $400 \mathrm{mg}$ IV TDS. After the diagnosis of ruptured hydatid cyst was made T. Albendazole $400 \mathrm{mg}$ BD was added and a Thoracic surgery opinion was sought for surgical management of the ruptured cyst. The patient was later transferred to Thoracic Surgery ward for excision of the cyst.

\section{Discussion}

The liver is the most commonly affected organ (in $60 \%$ of cases), and the lungs are affected in $20 \%$ to $30 \%$ of cases. In pulmonary hydatid disease, the cyst can be anywhere in the lungs but has a predilection for the right lung (54\% to $63 \%$ right vs. $37 \%$ to $46 \%$ left) and for the lower lobes (52\% to $67.5 \%$ ). In this case we had a single hydatid cyst involving the left upper lobe without involvement of the liver. Also because of the presence of an air fluid level this case was initially diagnosed as a case of lung abscess by the radiologist. But the left upper lobe is an uncommon site for both a lung abscess and a hydatid cyst. Therefore we decided to further investigate the case with a flexible video bronchoscopy and found the presence of membranous tissue obstructing the left upper lobe bronchus going in favour of a ruptured hydatid cyst. A review of the CT report following this finding was concluded as a ruptured hydatid cyst with surrounding consolidation. A complicated hydatid cyst can cause unusual radiological features on USG, CT and MRI leading to misdiagnosis. ${ }^{3}$ A similar case of a ruptured pulmonary hydatid cyst with complete collapse of left lung with pyopneumothorax and bronchopleural fistula misdiagnosed as pulmonary tuberculosis was reported by Puri D et al. ${ }^{4}$ Another case of a 22 -year-old woman with atypical clinical and radiological presentation was found to have a necrotic looking endobronchial lesion 
completely obstructing the superior segment of the left lower lobe on bronchoscopy which was proven to be a hydatid cyst on histopathology. ${ }^{5}$ Therefore bronchoscopy should be considered as an important investigation to differentiate between an unusually located hydatid or lung abscess with atypical clinical and radiological features.

\section{References}

1. Gottstein B, Reichen J. Hydatid lung disease (echinococcosis / hydatidosis). Clin Chest Med 2002;23:397-408.

2. Morar R, Feldman C. Pulmonary echinococcosis. Eur Respir J 2003;21:1069-77.

3. Turgut AT, Altın L, Topçu S, Kılıçoğlu B, Altınok T, Kaptanoğlu E, et al. Unusual imaging characteristics of complicated hydatid disease. Eur J Radiol 2017 Jul 4;63(1):84-93.

4. Puri D, Mandal AK, Kaur HP, Mahant TS. Ruptured hydatid cyst with an unusual presentation. Case Rep Surg 2011; 2011:730604.

5. Sharif A, Ansarin K, Rashidi F, Taghizadieh A. Bronchoscopic diagnosis and removal of a ruptured hydatid cyst. J Bronchol Interv Pulmonol 2011;18(4):362-4. 